\section{Therapy with tyrosine kinase inhibitor lenvatinib in radioactive iodine-naive advanced differentiated thyroid cancer}

\author{
Jasmine Singh Sukumar ${ }^{1}$, William Moore ${ }^{2}$ \& Saad A Khan*,3,4 \\ ${ }^{1}$ Divisions of Hematology and Medical Oncology, The Ohio State University Medical Center, Columbus, OH 43210, USA \\ ${ }^{2}$ Department of Radiology, UT Southwestern Medical Center, Dallas, TX 75390, USA \\ ${ }^{3}$ UT Southwestern Department of Internal Medicine and Harold C Simmons Comprehensive Cancer Center, Dallas, TX 75390, USA \\ ${ }^{4}$ MD, UT Southwestern Medical Center, Dallas, TX 75390-8852, USA \\ *Author for correspondence: Saad.Khan@UTSouthwestern.edu
}

First draft submitted: 17 June 2019; Accepted for publication: 3 October 2019; Published online: 7 November 2019

Keywords: differentiated thyroid cancer $\bullet$ lenvatinib $\bullet$ papillary thyroid cancer $\bullet$ radioactive iodine-naive $\bullet$ tyrosine kinase inhibitor

Lenvatinib is a tyrosine kinase inhibitor (TKI) approved for the management of radioactive iodine (RAI) refractory differentiated thyroid cancer (DTC) [1]. In the Phase III SELECT trial, lenvatinib extended median progression free survival to 18.3 months compared with 3.6 months for placebo and a response rate of $64.8 \%$ [2]. This trial required that enrolled patients have previously received and deemed refractory to RAI. There are no published data on the efficacy of TKIs in patients who have not previously received RAI and it is unclear if the molecular changes that thyroid cancer undergoes after exposure to RAI are critical for response to lenvatinib.

RAI is an established adjuvant treatment for high-risk patients who have undergone surgical resection and in those that have recurrent or metastatic disease. However, the 2015 American Thyroid Association (ATA) guidelines [3] do not address the role of RAI prior to thyroidectomy, aisutionat that commonly arises in clinical practice. Patients that present with locoregionally advanced DTC initially are offered drastic surgical options that may be declined due to patient- or tumor-specific factors. Extremely large tumors may require a laryngectomy which can negatively impact quality of life and increase morbidity [4]. Also, these patients may not be ideal candidates for RAI as RAI may be less likely to concentrate in patients with large tumors and may not effectively reduce tumor burden [5]. There are limited data on the impact of preoperative RAI [6], though two recent reports described 'neoadjuvant' lenvatinib without prior RAI [7-9]. Both cases resulted in dramatic tumor reduction and were followed by surgery and post-lenvatinib RAI. Preoperative or palliative radiation therapy (with or without systemic therapy) is widely recognized as leading to increased complication rates after head and neck cancer surgery, especially if reconstruction is required [10-14]. However, it is not universally effective and additional treatment options are needed for patients with large thyroid cancers who are unlikely to benefit from RAI due to their large tumor burden.

Patients who present with large thyroid cancers and decline surgery due to concerns about functional outcome present distinct challenges. Any decrease in tumor size with systemic therapy may reduce the extent of surgical interventions such as laryngectomy, tracheal resection and esophageal-pharyngeal resection in favor of less radical surgical approaches. Thus, even though the approved indication for lenvatinib is for patients with radioactive iodine refractory thyroid cancer, early systemic therapy may be particularly beneficial in patients with extensively invasive DTC or more aggressive histologies such as poorly differentiated thyroid cancer. While tumor shrinkage may be expected with preoperative lenvatinib, it is unknown what other impact this may have; whether the anti-angiogenic effects of lenvatinib complicate surgery, whether pathologic evidence of lymph node is impaired or if outcomes are improved. Ongoing clinical trials may help answer some of these questions (NCT03506048).

Lenvatinib use should not be routinely expanded out to patients before they receive RAI. However, in practices such as ours where patients present with extremely large bulky thyroid cancers and the only surgical option is a laryngectomy followed by RAI, our experience suggests that treating such patients with lenvatinib prior to any RAI therapy yields durable disease control and opens up additional treatment options. Our novel results suggest
Future $\because$ Medicine 


\begin{tabular}{|c|c|c|c|c|c|c|}
\hline Age & Race & Sex & Primary site & $\begin{array}{l}\text { Treatment prior to } \\
\text { lenvatinib }\end{array}$ & Lenvatinib toxicity & Best response to lenvatinib \\
\hline 69 & Caucasian & M & $\begin{array}{l}\text { Left thyroid with } \\
\text { extension to trachea and } \\
\text { superior mediastinum }\end{array}$ & $\begin{array}{l}\text { 1. Total thyroidectomy } \\
\text { with left paratracheal } \\
\text { dissection } \\
\text { 2. External beam radiation } \\
70 \text { Gy }\end{array}$ & $\begin{array}{l}\text { Myalgias, dysgeusia, } \\
\text { weight loss }\end{array}$ & $\begin{array}{l}\text { Partial response: } \\
- \text { Reduction in paratracheal mass } \\
4.3 \times 1.4 \rightarrow \\
1.0 \times 1.0 \mathrm{~cm} \\
- \text { Followed by radioactive iodine and } \\
\text { no resumption of lenvatinib for } \\
>12 \text { months (ongoing) }\end{array}$ \\
\hline 69 & Hispanic & $\mathrm{F}$ & $\begin{array}{l}\text { Left thyroid with invasion } \\
\text { to trachea and esophagus, } \\
\text { cervical and mediastinal } \\
\text { lymph nodes, lung }\end{array}$ & None & $\begin{array}{l}\text { Hypertension, neuropathy, } \\
\text { myalgias, weight loss }\end{array}$ & $\begin{array}{l}\text { Partial response: } \\
\text { - Decrease in size of bilateral } \\
\text { pulmonary nodules, mediastinal } \\
\text { lymph nodes, and thyroid mass, } \\
\text { improvement in malignant pleural } \\
\text { effusion } \\
\text { - Followed by radioactive iodine } \\
\text { (suboptimal response), remains on } \\
\text { lenvatinib }\end{array}$ \\
\hline
\end{tabular}

lenvatinib therapy is active in patients with bulky thyroid cancer who require major surgery, even those who have never previously been treated with RAI. The goal of this approach is to delay surgery or make it unnecessary, enhance the chance of cure and provide more treatment options for patients. We present a report of two patients who had not received any prior RAI when they were treated with lenvatinib (Table 1).

\section{Patient}

Patient 1

A 69-year-old Caucasian male presented with 6 months of dyspnea and, in August 2016, a computed tomography (CT) neck scan showed enlargement of the left thyroid with extension into the superior mediastinum and rightward tracheal deviation. Thyroid ultrasound revealed a multinodular thyroid with a large exophytic left lobe nodular conglomerate (approximately $9.3 \times 4.3 \times 7.1 \mathrm{~cm}$ ) and biopsy showed papillary thyroid carcinoma.

He underwent a total thyroidectomy with left paratracheal dissection with median sternotomy in September 2016. Residual disease was left on the anterior tracheal wall, left cricotracheal junction and right thyroid bed. The pathology returned as papillary thyroid carcinoma, classic type with predominantly follicular and focal trabecular growth pattern (up to $12 \mathrm{~cm}$ ), with positive margins, lymphovascular invasion and extrathryoidal extension. There was also microscopic papillary thyroid carcinoma $(0.1 \mathrm{~mm})$ in the right thyroid lobe. He was started on levothyroxine suppression. His surgical recovery was complicated by vocal cord paresis, severe dysphagia and aspiration with subsequent gastrostomy tube placement.

He received adjuvant external beam radiation therapy to the neck and superior mediastinum with a total of 70 Gy from November to December 2016. After radiation, he developed improved swallow function and voice quality. Positron Emission Tomography in April 2017 showed paraesophageal mass in the thyroidectomy bed of $4.3 \times 1.4 \mathrm{~cm}$ consistent with recurrence and fine needle aspiration revealed recurrent papillary thyroid cancer. He was offered surgical intervention with total laryngectomy but he did not want to pursue this. He was assessed by endocrinology for RAI administration. Due to the fluorodeoxyglucose (FDG) avidity and bulkiness of the tumor as well as logistical difficulty of administering RAI in a gastrostomy tube-dependent patient, he was not administered RAI because these features suggested the tumor would be RAI resistant. He was thus started on lenvatinib $20 \mathrm{mg}$ daily in May 2017, which would be considered an off-label use as he had not received any prior RAI. Thyroglobulin was $20 \mathrm{ng} / \mathrm{ml}$ after radiation was completed and then dropped to $1 \mathrm{ng} / \mathrm{ml}$ and $0.6 \mathrm{ng} / \mathrm{ml}$ after 2 and 3 months of lenvatinib respectively.

A CT neck and chest scan in July 2017 showed reduction in size of paratracheal mass, demonstrating favorable response to TKI. Lenvatinib was held in August 2017, after the patient developed an axillary abscess, which was treated. He also developed myalgias and dysgeusia which he attributed to the medication. Repeat positron emission tomography in September showed decreased side of paraesophageal mass to $2.6 \times 1 \mathrm{~cm}$. He was restarted on lenvatinib in October 2017 at $10 \mathrm{mg}$ daily. The patient was again offered surgery but declined both a mass resection and laryngectomy due to his concern about potential damage to his voice. Lenvatinib was continued for a total of 14 months until the size of the mass reduced to $1.1 \mathrm{~cm}$ in the longest dimension. At that time, he was administered $127 \mathrm{mCi}$ radioactive iodine (I-131) after thyrogen stimulation, with a subsequent scan showing no uptake in 
the neck. He then resumed taking his leftover supply of lenvatinib $10 \mathrm{mg}$ for 3 weeks. He had not been taking lenvatinib or any other anticancer treatment for 2 months when he had repeat imaging of this area performed. A CT scan of the area was described as being 'without evidence of residual/recurrent disease', with personal review of images identifying a $1 \times 1 \mathrm{~cm}$ mass in that area. The patient reported no symptoms of pain or residual toxicity. Thyroglobulin at that time increased to 3.7 from 1.8 prior to RAI.

\section{Patient 2}

A 69-year-old Hispanic female presented with persistent hoarseness and dysphagia for 1 year. Her past medical history included chronic lymphocytic leukemia, for which she received whole body radiation therapy and stem cell transplant in 2009 but was now in remission. Thyroid ultrasound in June 2016 showed a left thyroid lobe mass of $4.1 \times 2.3 \times 3.7 \mathrm{~cm}$, lymphadenopathy in the bilateral jugular chains and calcification of the right posterior midpole. A CT neck scan showed the left mass to be $3.1 \times 2.8 \mathrm{~cm}$ and biopsy revealed papillary thyroid carcinoma. A CT chest scan showed multiple pulmonary nodules and mediastinal lymphadenopathy suspicious for metastasis. A repeat CT chest scan in August 2016 showed progression of known left thyroid papillary carcinoma with concern for invasion of the trachea and esophagus with left vocal cord paralysis. Her bulky tumor was considered as likely resistant to RAI based on the 2015 ATA Guidelines and therefore RAI was felt to be of minimal benefit in causing rapid tumor shrinkage.

The patient was started on lenvatinib with a goal to shrink tumor size prior to surgical intervention, which would require laryngectomy if pursued early. She started lenvatinib $24 \mathrm{mg}$ daily in August 2016 but subsequently developed hypertension. Lenvatinib was decreased to $14 \mathrm{mg}$ daily, which she received for 2 weeks in August 2016, but given persistent hypertension, it was then held. In September, lenvatinib was restarted at $10 \mathrm{mg}$ daily and was further reduced to $8 \mathrm{mg}$ daily in November due to neuropathy and myalgias. A repeat CT chest scan in October 2016 demonstrated favorable response to treatment, including decrease in size of bilateral pulmonary nodules, mediastinal lymphadenopathy and left thyroid mass. MRI of the neck showed persistent invasion of the left trachea, left vocal cord and esophagus.

From March to April 2017, lenvatinib was held and she received intensity-modulated radiotherapy of 60 Gy in 25 fractions to the left thyroid and neck over a duration of 5 weeks. In September 2017, she received RAI with 129 millicuries of I-131 after thyrogen stimulation. She was then started on levothyroxine suppression. Subsequent radioiodine whole body scan revealed evidence of lung metastases with poor response to iodine uptake. Lenvatinib was resumed at $8 \mathrm{mg}$ daily in October 2017 but then stopped in November due to dyspnea and hypertension. A CT chest scan showed multifocal areas of nodular pleural thickening worrisome for pleural metastases with moderate sized left pleural effusion. Her blood pressure was controlled with antihypertensive medications. Thoracentesis improved symptoms and cytology was consistent with malignant pleural effusion. Lenvatinib was restarted at $8 \mathrm{mg}$ daily in December 2017. Given reaccumulating nature of pleural effusion indwelling intrapleural catheter was placed and she received pleurodesis in December 2017. Catheter was removed in February 2018 given improvement in pleural effusion. She had progressive weight loss and, in March 2018, lenvatinib was decreased to $4 \mathrm{mg}$ daily. Imaging in June 2019 shows sustained stability of disease.

\section{Discussion}

The two presented cases demonstrate that lenvatinib can play a major role in disease control in patients who have never previously received RAI. Our findings are consistent with that of Danilovic et al., who described a patient with unresectable papillary thyroid carcinoma, in whom preoperative treatment with TKI sorafenib allowed for reduction in tumor volume, leading to new treatment options, including surgical intervention via thyroidectomy and RAI [15]. Two other published reports have described lenvatinib responses in differentiated thyroid cancer patients who have not received prior RAI [7-9]. Similar outcomes have also been observed with neoadjuvant targeted systemic therapy for anaplastic thyroid cancer [16].

To be clear, such usage of lenvatinib is not considered standard practice nor is it one of the indications for use in its regulatory approval. Practices such as ours regularly see patients with large thyroid cancers who may not be candidates for, or decline, surgery, radiation or RAI. These patients still require treatment and the tolerability and anticancer efficacy of lenvatinib make it an attractive option. In cases where the likelihood of RAI without drastic surgery being effective is low to zero, administering it before lenvatinib may be the incorrect option even if it is considered the standard option. While laryngectomy followed by RAI for residual disease may indeed be the correct option for many patients, some patients with a curative option that mandated laryngectomy were more receptive 


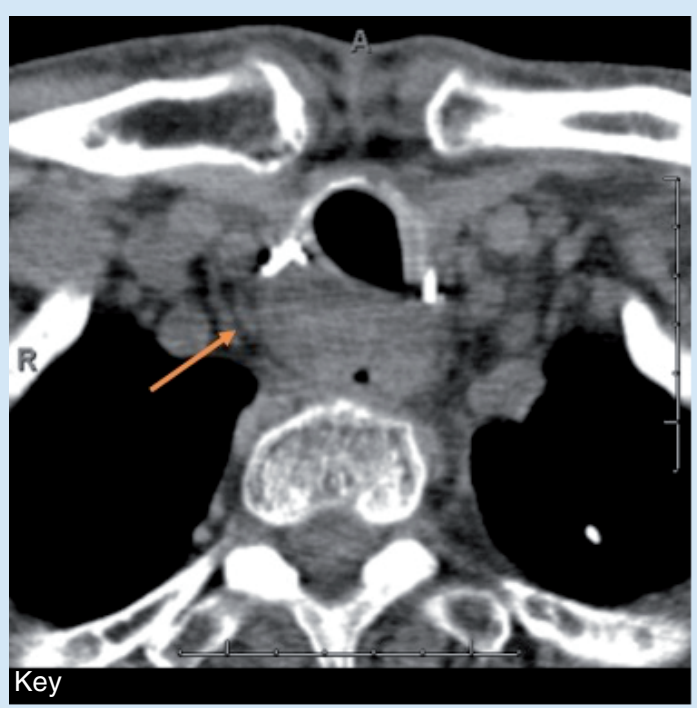

April 2017

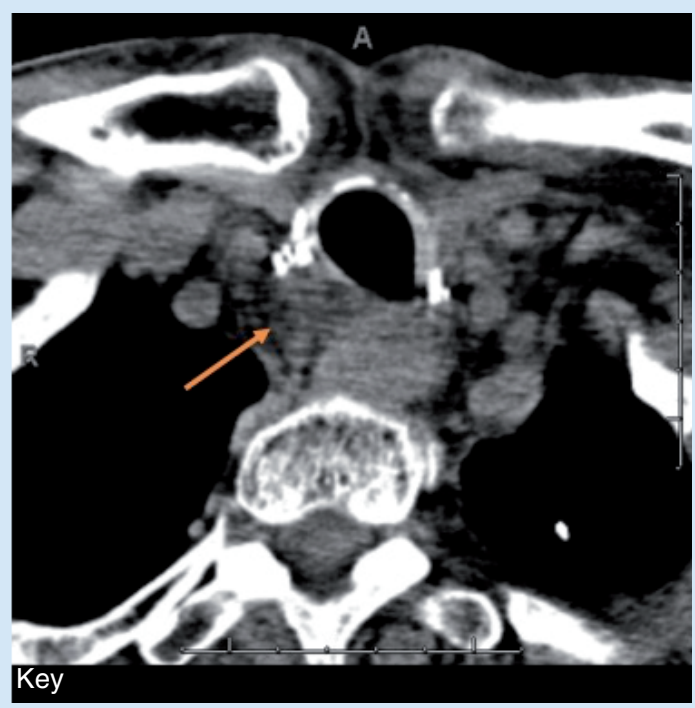

September 2017

Figure 1. Axial imaging showing tumor response after 6 months of lenvatinib, without prior radioactive iodine.

to disease control with medication. The decision against surgery in favor of lenvatinib is based on the perceived improved life with retaining a functioning larynx, even if it comes at the expense of a theoretically shorter lifespan with noncurative lenvatinib.

In these two patients, lenvatinib showed excellent activity in RAI-naive patients, but did not result in converting a radical surgery into a simpler procedure. In one patient, there also did not appear to be significant activity of RAI in these patients after lenvatinib administration; this suggests that lenvatinib may differ from some other TKI's that enhance RAI sensitivity. Though formal quality of life assessments were not performed, both patients reported tolerating lenvatinib well with standard dose reductions and no significant periods of treatment interruption. They remained very anxious about possible surgical complications. Patient 1 was able to tolerate regular dosing for 14 months and resumed it even when he was not instructed to do so. The tumor mass reduced in size from over $4 \mathrm{~cm}$ in longest dimension to $1 \mathrm{~cm}$ in size just after 6 months of regular dosing with lenvatinib (Figure 1). Although laryngectomy was offered to the patient for potential cure, he declined given concern for adverse effects related to surgery. Therefore, the degree of anticancer activity was highly effective for this patient for the amount of toxicity faced, even if it was not yet a curative option. The second patient developed metastatic pleural effusion and required frequent dose interruptions due to existing poorly controlled blood pressure. However, even with intermittent or decreased dosing, there were favorable effects. In Patient 2, the pleural effusion completely resolved, resulting in significantly improved quality of life (Figure 2). The side effect of lenvatinib in our patients was similar to that seen in RAI refractory patients.

Our patients also demonstrate an interesting aspect about lenvatinib. The dramatic reduction in the volume seen with lenvatinib meant that RAI became an option for therapy. Generally, RAI is effective for low volume DTC that may be localized or metastatic. RAI was initially not recommended for Patient 2 due to the volume of the cancer as well as lack of prior thyroidectomy. However, as the visible burden of disease markedly reduced, both patients received RAI therapy without uptake in their thyroidectomy bed. Lenvatinib is thought to exert its antithyroid cancer effect via VEGF, but it does inhibit MEK as well [10]. MEK inhibitors such as Selumetinib and Trametinib can lead to RAI sensitivity in patients that were previously RAI refractory [11]. It is posited that lenvatinib may lead to enhanced sensitivity to RAI, but in these two patients, this effect is not observed. Whether lenvatinib results in enhanced RAI uptake is currently unclear but the subject of ongoing clinical trials (NCT03506048; PI D Taofeek K/Owonikoko).

Lenvatinib generally has a rapid time to tumor response, that is one reason why it may be a particularly effective agent in patients with large tumors that may not respond to RAI. In the SELECT trial, it was shown to have a 


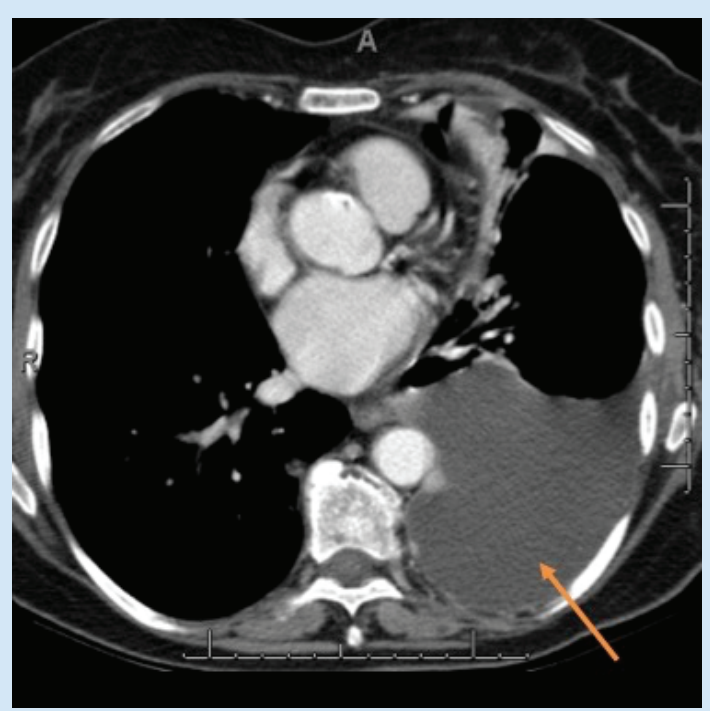

September 2017

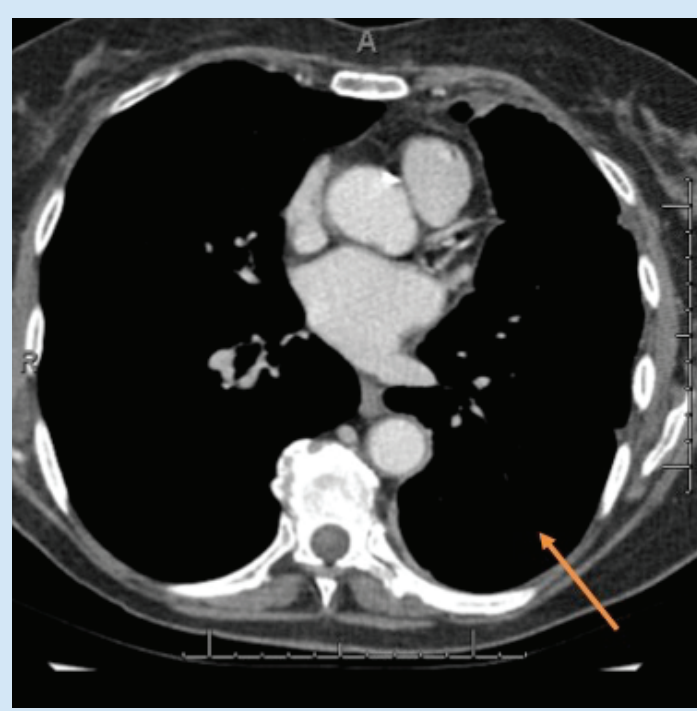

February 2018

Figure 2. Axial imaging showing dramatic reduction in pleural effusion after 5 months of lenvatinib without prior radioactive iodine.

median time for an objective response of 2 months (95\% CI: 1.9-3.5) [2]. The dramatic degree of response seen in our patients suggests that the mechanism of action remains the same in patients with DTC that have never been exposed to RAI.

In conclusion, our experience suggests that lenvatinib is effective in DTC that has never been treated with RAI. Early lenvatinib may be a novel approach to convert an unresectable case into one where a more limited surgery is possible, though patients who tolerate lenvatinib well may still remain hesitant to consider any surgery. Lenvatinib reduced the volume of thyroid cancer that had never been treated with RAI to such a degree that subsequent therapy with RAI became possible. In patients who decline surgical resection, have bulky tumors and have other findings suggestive of RAI refractoriness, lenvatinib should not be held for the sole reason that RAI has yet to be administered. The authors caution that these very narrow clinical scenarios detailed in this manuscript should not be used to justify indiscriminate use of lenvatinib in patients who appropriately meet ATA guidelines for surgical resection followed by RAI. Future prospective clinical trials will define lenvatinib's role as a costeffective, safe and efficacious treatment in the general patient population who have not been treated with RAI previously (e.g., NCT03506048).

\section{Ethical conduct of research}

Informed consent for the use of patient details was obtained from both patients and is present in the medical record. Both patients freely provided this consent to the treating team with the only limitation of no identifiable information being included in the manuscript as well as a request for the final published version. Institutional review board oversight is thus not required as both patients were not treated with research intent. The authors state that they have obtained verbal and written informed consent from the patient/patients for the inclusion of their medical and treatment history within this case report.

Financial \& competing interests disclosure

The authors have no relevant affiliations or financial involvement with any organization or entity with a financial interest in or financial conflict with the subject matter or materials discussed in the manuscript. This includes employment, consultancies, honoraria, stock ownership or options, expert testimony, grants or patents received or pending, or royalties.

No writing assistance was utilized in the production of this manuscript. 
Open access

This work is licensed under the Attribution-NonCommercial-NoDerivatives 4.0 Unported License. To view a copy of this license, visit http://creativecommons.org/licenses/by-nc-nd/4.0/

\section{References}

Papers of special note have been highlighted as: $\bullet$ of interest; $\bullet \bullet$ of considerable interest

1. Schmidt A, Iglesias L, Klain M, Pitoia F, Schlumberger MJ. Radioactive iodine-refractory differentiated thyroid cancer: an uncommon but challenging situation. Arch. Endocrinol. Metab. 61(1), 81-89 (2017).

2. Schlumberger M, Tahara M, Wirth LJ et al. Lenvatinib versus placebo in radioiodine-refractory thyroid cancer. N. Engl. J. Med. 372(7), 621-630 (2015).

3. Haugen BR, Alexander EK, Bible KC et al. 2015 American Thyroid Association management guidelines for adult patients with thyroid nodules and differentiated thyroid cancer: the American Thyroid Association guidelines task force on thyroid nodules and differentiated thyroid cancer. Thyroid 26(1), 1-133 (2016).

- Guidelines for use of radioactive iodine and lenvatinib in thyroid cancer.

4. Ark N, Zemo S, Nolen D, Holsinger FC, Weber RS. Management of locally invasive well-differentiated thyroid cancer. Surg. Oncol. Clin. N. Am. 17(1), 145-155 (2008).

5. Vassilopoulou-Sellin R, Schultz PN, Haynie TP. Clinical outcome of patients with papillary thyroid carcinoma who have recurrence after initial radioactive iodine therapy. Cancer 78(3), 493-501 (1996).

6. Shingu K, Kobayashi S, Yokoyama S et al. Effectiveness of preoperative radioactive iodine (131I) therapy for locally advanced papillary thyroid cancer: a case report. Thyroid 8(12), 1113-1116 (1998).

7. Tsuboi M, Takizawa H, Aoyama M, Tangoku A. Surgical treatment of locally advanced papillary thyroid carcinoma after response to lenvatinib: a case report. Int. J. Surg. Case Rep. 41, 89-92 (2017).

-. Other examples of preoperative lenvatinib use that was effective.

8. Stewart KE, Strachan MWJ, Srinivasan D, MacNeill M, Wall L, Nixon IJ. Tyrosine kinase inhibitor therapy in locally advanced differentiated thyroid cancer: a case report. Eur. Thyroid J. 8(2), 102-107 (2019).

-. Other examples of preoperative lenvatinib use that was effective.

9. Sheu NW, Jiang HJ, Wu CW, Chiang FY, Chiou HC, Hsiao PJ. Lenvatinib complementary with radioiodine therapy for patients with advanced differentiated thyroid carcinoma: case reports and literature review. World J. Surg. Oncol. 17(1), 84 (2019).

10. Terezakis SA, Lee KS, Ghossein RA et al. Role of external beam radiotherapy in patients with advanced or recurrent nonanaplastic thyroid cancer: memorial Sloan-kettering cancer center experience. Int. J. Radiat. Oncol. Biol. Phys. 73(3), 795-801 (2009).

11. Cortes-Mateus KS, Holub K, Racca F, Grau JJ, Capdevila J. Concurrent palliative external radiotherapy with sorafenib or doxorubicin for bulky differentiated thyroid carcinoma: a case report. Oncol. Lett. 16(3), 4085-4089 (2018).

12. Herle P, Shukla L, Morrison WA, Shayan R. Preoperative radiation and free flap outcomes for head and neck reconstruction: a systematic review and meta-analysis. ANZ J. Surg. 85(3), 121-127 (2015).

13. Sokoya M, Bahrami A, Vincent A et al. Preoperative radiation and complication rates after double free flap reconstruction of head and neck cancer. Am. J. Otolaryngol. 39(5), 558-560 (2018).

14. Righini CA, Nadour K, Faure C et al. Salvage surgery after radiotherapy for oropharyngeal cancer. Treatment complications and oncological results. Eur. Ann. Otorhinolaryngol. Head Neck Dis. 129(1), 11-16 (2012).

15. Danilovic DLS, Castro G Jr, Roitberg FSR et al. Potential role of sorafenib as neoadjuvant therapy in unresectable papillary thyroid cancer. Arch. Endocrinol. Metab. 62(3), 370-375 (2018).

16. Cabanillas ME, Ferrarotto R, Garden AS et al. Neoadjuvant BRAF- and immune-directed therapy for anaplastic thyroid carcinoma. Thyroid 28(7), 945-951 (2018). 